\title{
A Compliant Contact-aided Revolute Joint
}

Jessie R. Cannon

Larry L. Howell

lhowell@byu.edu

Follow this and additional works at: https://scholarsarchive.byu.edu/facpub

Part of the Mechanical Engineering Commons

\section{Original Publication Citation}

Cannon, J.R. and Howell, L.L., "A Compliant Contact-aided Revolute Joint," Mechanism and Machine Theory, Vol. 4, No. 11, pp. 1273-1293, 25.

\section{BYU ScholarsArchive Citation}

Cannon, Jessie R. and Howell, Larry L., "A Compliant Contact-aided Revolute Joint" (2005). Faculty Publications. 341.

https://scholarsarchive.byu.edu/facpub/341 accepted for inclusion in Faculty Publications by an authorized administrator of BYU ScholarsArchive. For more information, please contact ellen_amatangelo@byu.edu. 


\title{
A compliant contact-aided revolute joint
}

\author{
Jesse R. Cannon, Larry L. Howell* \\ Department of Mechanical Engineering, Brigham Young University, P.O. Box 24102, Provo, UT 84602, USA
}

\begin{abstract}
This paper presents the compliant contact-aided revolute (CCAR) joint, a planar mechanism capable of performing the functions of a bearing and a spring. The pseudo-rigid-body model is used to predict the behavior of the CCAR joint, and this model is validated through the use of finite element analysis and prototype testing. The CCAR joint is shown to have high maximum rotation and lateral stiffness. A case study is presented, and manufacturing considerations are discussed for the macro, meso, and micro scales.
\end{abstract}

Keywords: Compliant mechanisms; Revolute joints; Bearings; Springs; Contact interactions

\section{Introduction}

Bearings and helical springs are traditional mechanical elements commonly used in machine design. Helical springs are used to provide energy storage or reaction to a force or torque. Bearings use either sliding contact or rolling elements to constrain lateral translation while allowing rotation between two rigid parts. Sliding contact bearings such as bushings are composed of as few as two parts, making them simple to manufacture and assemble. They can have high energy loss, however, due to friction between the sliding surfaces. Rolling-element bearings have much lower friction, yet typically have from 10 to 30 parts,

\footnotetext{
* Corresponding author. Tel.: +1-801-422-8037; fax: +1-801-422-5037.

E-mail address: 1howell@byu.edu (L.L. Howell).
} 
increasing manufacturing costs and assembly time. Both types of bearings usually need lubrication to reduce friction and remove heat. They can also suffer from backlash [1].

An increasing number of mechanical systems are being designed on the micro and meso scales, but the minimum size of traditional bearings and helical springs is limited. Fabrication methods at these scales are usually limited to planar geometries, and assembly and maintenance become increasingly difficult as the size of mechanisms decrease. The backlash of bearings also limits their usefulness in applications where high precision and repeatability are needed. At small scales and for high precision applications, alternative, nontraditional elements are needed.

These non-traditional elements should retain the functions of their traditional counterparts. To perform the function of a helical spring, a component must be able to produce specified torque/motion requirements. Components performing the function of a bearing must permit sufficient motion about the axis of rotation, bear specified loads in lateral directions, and fit within roughly the same design space as a bearing.

\section{The compliant contact-aided revolute joint}

The compliant contact-aided revolute (CCAR) joint, shown in Figure 1, is described in this paper as a means of addressing the needs discussed above. The CCAR joint consists of multiple flexures, fixed at one end (Point B), and free at the other (Point A). The free ends of the flexures are constrained by the cam surface of the gauge pin. The cam surface has a constant radius of curvature, $R_{c}$, with its center located at the Point C. The contact surfaces also have a constant radius of curvature, $R_{s}$, and interact with the flexures. Deflection of the flexures allows the CCAR joint to rotate, and provides a restoring torque. As the flexures deflect they come into contact with the contact surfaces. The contact surfaces constrain the flexures to have a constant radius of curvature along the contacting segments of each flexure. The geometry of the CCAR joint is planar, allowing it to be manufactured at smaller scales using planar fabrication methods.

The number of flexures used in a CCAR joint depends primarily on the rotation and the torque characteristics desired for the design. The maximum possible rotation of the joint increases as the radius of the cam surface, $R_{c}$, increases. If $R_{c}$ is too large, however, the number of flexures must decrease, 
and fewer flexures will decrease the torque. A general guideline, therefore, is that more flexures should be used for CCAR joint designs requiring high torque and small rotation, and fewer flexures for designs requiring low torque and large rotation.

This paper gives a review of existing compliant revolute joints, and discusses principles that are used to achieve enhanced performance for the CCAR joint. Models for predicting the behavior of the CCAR joint are developed, and methods for its manufacture discussed. Finally, a case study for the application of the CCAR joint is presented.

\section{Backround}

\subsection{Compliant revolute joints}

Compliant mechanisms gain at least some of their mobility from the deflection of flexible members [2] instead of from the relative motion between separate, rigid parts. They can often be made in one piece, reducing the part count of a mechanism, simplifying its assembly requirements, and making them easy to miniaturize. Compliant mechanisms are also capable of reducing or eliminating the friction, wear, and backlash associated with traditional joints.

Compliant components that perform the function of bearings fall under a class of mechanisms known as compliant revolute joints. Moon et al. [3] have suggested that five criteria be used in comparing different types of compliant revolute joints: (1) range of motion, (2) amount of axis drift, (3) ratio of off-axis stiffness to stiffness about the axis of rotation, (4) stress concentration effects, and (5) compactness of the joint. They also provided a qualitative comparison of some of the existing compliant revolute joints, rating each joint as 'poor', 'normal', or 'good' for each of the five criteria.

Notch-type joints were first described by Paros and Weisbord [4]. Methods that use leaf springs to create a revolute joint are described in [5-8], and methods using circular leaf springs are described in [7]. The split-tube flexure introduced by Goldfarb and Speich [9] and the cross-type joint introduced by Moon et al. [3] gain their motion from deflection of elements in torsion.

To replace a bearing, a compliant component should permit a large range of motion about the axis of rotation, have a high stiffness in lateral directions, and be compact enough to fit within the same design space 
as a bearing. These objectives correspond to the criteria 1,4 , and 5 of Moon et al. The only joints that are rated as at least 'normal' or above in all three of these criteria are the two torsional joints in [3] and [9], and none of them are rated as 'good' in all three.

\subsection{Contact-aided compliant mechanisms}

Contact interactions can be used to improve the performance of a compliant revolute joint. Mankame and Ananthasuresh [10] have shown that contact-aided compliant mechanisms-compliant mechanisms that use contact interactions with external bodies or with different parts of the mechanism itself-can have enhanced functionality when compared with traditional compliant mechanisms. The flexible members used in traditional compliant mechanisms are typically constrained by boundary conditions at their ends. Examples include a cantilever beam with a force at the free end, a beam fixed at one end and guided at the other, or a beam that is pinned at both ends [2]. Beams that contact other elements along their length have the potential of creating favorable behaviors that might not be achieved by beams constrained only at their ends.

Contact-aided compliant mechanisms can have an improved ability to bear lateral loads. Guérinot [11] has demonstrated that the contact of rigid elements in a joint can divert a compressive load away from buckling-prone flexible segments. Contact interactions can also increase the maximum possible deflection of a compliant beam [12].

Chironis and Sclater [13] presented a concept where a cantilever beam is placed adjacent to a constant radius surface. As the beam deflects, a portion of the beam comes into contact with the surface. This type of contact interaction increases both the deflection of the beam and the beam's resistance to buckling. The design introduced in this paper uses a similar concept to increase the rotation and lateral stiffness of a compliant revolute joint.

\section{Modeling}

As the CCAR joint rotates, the flexures come into contact with the opposing contact surfaces. Figure 2 represents a flexure in contact with a constant radius surface. The length of beam in contact with the surface is measured by the variable $\theta_{s}$, which increases with the rotation of the joint. This contact interaction will be 
developed by first analyzing the contacting segment of the beam, and then analyzing the non-contacting segment.

\subsection{Contacting segment analysis}

Contact of the beams with the surfaces creates well defined moments in the beams due to the relationship between the radius of curvature of a beam and the moment. This relationship is derived from the Bernoulli-Euler equation, which states

$$
M=E I \frac{d \theta}{d s}
$$

where $E$ is the modulus of elasticity, and $I$ is the moment of inertia of the cross section. $\frac{d \theta}{d s}$ is the curvature, defined as the rate of change of the angle of the beam, $\theta$, per unit of arc length, $s$. Defined in this way, the Bernoulli-Euler equation is valid for large deflections. Assumptions made when using equation (1) are that 1) the material is linearly elastic, homogeneous, and isotropic, 2) the transverse-shear component of deflection is small compared to that due to bending [1], and 3) the beams are thin, so that the neutral axis can be assumed to be coincident with the centroidal axis even for initially curved beams [2]. The effective radius of curvature of the beam is equal to

$$
R^{\prime}=\frac{1}{d \theta / d s}
$$

Some beams may have an initial curvature in their undeflected state. The effective radius of curvature takes into account any initial curvature of the beam using the equation

$$
R^{\prime}=\left(\frac{1}{R_{s}}-\frac{1}{R_{0}}\right)^{-1}
$$

where $R_{s}$ is the radius of curvature of the surface constraining the beam's shape, $R_{0}$ is the beam's initial radius of curvature, and the beam's thickness is assumed to be small compared to $R_{s}$. If the beam is initially straight $\left(R_{0}=\infty\right)$ then 


$$
R^{\prime}=R_{s}
$$

Substituting equation (4) into equation (1) gives

$$
M=\frac{E I}{R^{\prime}}
$$

When a beam is in contact with a surface that has a constant radius of curvature, the radius of curvature of the beam is also constrained to be constant, as in Figure 3(a). Equation (5) predicts that this constant radius of curvature throughout the beam will create a moment that is also constant along the beam length. This means the segment has the same behavior as a beam with a moment applied to the free end, as shown in Figure 3(b).

The maximum stress in a rectangular beam in bending is equal to

$$
\sigma_{\max }=\frac{M h}{2 I}
$$

where $h$ is the thickness of the beam. Because the moment is constant along the length of a beam in contact with a constant radius surface, equation (6) predicts that the stress will also be constant along its length. This constant stress is given by substituting equation (5) into equation (6):

$$
\sigma_{\max }=\frac{E h}{2 R^{\prime}}
$$

Thus the maximum stress in the CCAR joint depends only on the modulus of elasticity of the chosen material, the thickness of the flexure, and the effective radius of curvature.

Initially, as the CCAR joint begins to rotate, the moment at the base of the beam will be less than the moment in equation (5). In this condition, the beam acts as a traditional cantilever beam, with $\theta_{s}$ being equal to zero. Contact with the surface is initiated once the moment at the base of the beam becomes equal to the moment in equation (5), and the length of the contacting segment will increase with additional rotation after initial contact occurs. The contacting segment has the same behavior as a beam with a moment applied to the free end, as shown in Figure 4.

The maximum stress for the beam is found in the contacting segment, and can be found using equation (7). 


\subsection{Non-contacting segment analysis}

The non-contacting segment can be treated as if it were rigidly attached at its base to the contact surface, as shown in Figure 5, with the condition that the moment at the base of the non-contacting segment equal the moment in the contacting segment, as defined by equation (5). The non-contacting segment can be modeled using the pseudo-rigid-body model (PRBM) [2], as shown in Figure 5.

The PRBM is a method for analyzing systems that undergo large, nonlinear deflections. It treats a compliant segment as if it were two rigid links pinned together, with a torsional spring at the pinned joint. This approximation can give very accurate results, predicting the position of the end of the beam with an error of less than $0.5 \%$. The PRBM allows compliant mechanisms to be analyzed as if they were rigid-link mechanisms. Traditional rigid-link mechanism theory can be used to determine the motion and forcedeflection relationship of a compliant mechanism. More details on the PRBM can be found in [2].

The length of the non-contacting segment of the beam is

$$
l_{f}^{\prime}\left(\theta_{s}\right)=l_{f}-\theta_{s} R
$$

$l_{f}$ is the total length of the beam, including the contacting segment, and equals

$$
l_{f}=R_{r}+R_{c}-\delta_{c}-h / 2
$$

where $R_{r}$ and $\delta_{c}$ are geometric parameters defined in Figure 1(b).

The base of the non-contacting segment is at an angle of $\theta_{s}$ from the vertical, and the PRBM parameters $\phi$ and $\Theta$ are measured from this orientation. The position of the base is given by

$$
\begin{gathered}
x_{b}=(R) \sin \theta_{s} \\
y_{b}=(R)\left(1-\cos \theta_{s}\right)
\end{gathered}
$$

where $x_{b}$ and $y_{b}$ are the $x$ and $y$ positions as measured from the base of the contacting segment of the beam. 


\subsection{Kinematic analysis}

Using the pseudo-rigid-body model for the beam, the mechanism can be modeled as a rigid-body four-bar mechanism, as shown in Figure 6. The cam surface of the gauge pin can be modeled kinematically as if there were a link connecting Point A and Point C. This is link 4. Link 3 is the PRBM link of the noncontacting segment of the beam, and link 2 connects the PRBM link to the center of the mechanism. Both link 2 and link 3 change lengths as $\theta_{s}$ increases. Link 1 connects the center of the mechanism with the center of the cam surface arc. The following parameters are used in the derivation of the link lengths and angles, as shown in Figure 7:

$$
\begin{gathered}
\delta_{1}=\sqrt{\left(R_{r}-x_{b}\right)^{2}+y_{b}^{2}} \\
\delta_{2}=(1-\gamma) l_{f}^{\prime} \\
\alpha=\tan ^{-1}\left(\frac{y_{b}}{R_{r}-x_{b}}\right)
\end{gathered}
$$

The link lengths are given by the following equations:

$$
\begin{gathered}
r_{1}=\delta_{c} \\
r_{2}=\sqrt{\delta_{1}^{2}+\delta_{2}^{2}-2 \delta_{1} \delta_{2} \cos \left(\alpha+\theta_{s}\right)} \\
r_{3}=\gamma\left(l_{f}^{\prime}\right) \\
r_{4}=R_{c}-h / 2
\end{gathered}
$$

The angle $\theta_{2}$ is related to the total rotation of the mechanism, $\theta_{t}$, by the equation

$$
\theta_{2}=\theta_{t}+\alpha-\sin ^{-1}\left(\frac{\delta_{2}}{r_{2}} \sin \left(\alpha+\theta_{s}\right)\right)
$$

The angles $\theta_{3}$ and $\theta_{4}$ can be found using the closed-form equations for a four-bar mechanism. The force angle and pseudo-rigid-body angle are calculated as

$$
\begin{gathered}
\phi=\theta_{4}-\theta_{s}-\theta_{t} \\
\Theta=\theta_{3}-\theta_{s}-\theta_{t}-180
\end{gathered}
$$


and the torque experienced by the mechanism is

$$
T=n_{b} F \delta_{c} \sin \theta_{4}
$$

where $n_{b}$ is the number of beams in the mechanism, and $F$ is the force acting on the beam as predicted by the PRBM.

\subsection{Sensitivity analysis}

The output torque of the CCAR joint is a function of the flexure thickness, $h$, and the radius of the cam surface, $R_{c}$. The manufacturing tolerance is generally given as a surface profile tolerance [14], so maximum change in the radius of the cam surface, $\Delta R_{c}$, is equal to the tolerance. The flexure thickness, however, involves two surfaces; therefore the change in flexure thickness, $\Delta h$, is equal to twice the tolerance value.

The nominal condition is shown in Figure 8(a). The length of the flexure is decreased by twice the tolerance value creating a space between the flexure tip and the cam surface. This space in the nominal condition permits assembly of the CCAR joint in the upper bound condition (Figure 8(b)), the condition in which the torque will be highest. In this condition the flexure is thicker, the radius of the cam surface is smaller, and the space between the two parts is eliminated.

The upper bound for the torque is defined as

$$
T_{\text {upper }}=T\left(h_{\text {nom }}+\Delta h, R_{c_{\text {nom }}}-\Delta R_{c}\right)
$$

where $h_{n o m}$ is the nominal thickness of the flexure, $R_{c_{\text {nom }}}$ is the nominal radius of the cam surface. In the lower bound condition, shown in Figure 8(c), the flexure thickness is smaller, the radius of the cam surface is larger, and the space between the flexure tip and the cam surface is equal to four times the tolerance value. The lower bound for the torque is defined as

$$
T_{\text {lower }}=T\left(h_{\text {nom }}-\Delta h, R_{c_{\text {nom }}}+\Delta R_{c}\right)
$$

The space between the flexure tip and the cam surface results in zero torque during the first degrees of rotation. The flexure contacts the cam surface after the joint rotates $\theta_{t_{0}}$ degrees, where $\theta_{t_{0}}$ is defined as 


$$
\theta_{t_{0}}=\cos ^{-1}\left[\frac{r_{1}^{2}+\left(r_{2}-r_{3}\right)^{2}-r_{4}^{2}}{2 r_{1}\left(r_{2}-r_{3}\right)}\right]
$$

$r_{1}, r_{2}, r_{3}$, and $r_{4}$ are parameters in the kinematic model of the joint, and are functions of $h$ and $R_{c}$.

\subsection{Lateral load analysis}

The CCAR joint must be capable of withstanding lateral loads. When placed under lateral loads, some of the beams are placed into compression and must be able to support the load without buckling.

At maximum rotation, the contacting segment of the beam extends to Point $\mathrm{D}$ on the contact surface, as defined by the parameter $\theta_{s 2}$ (see Figure 1(b)). This parameter must be defined by the position of the flexure in the upper bound condition, since the flexure contacts the highest percentage of the surface in this condition. The profile of the contact surface after this point is no longer a constant radius, but is a curve defined by the linearized equation for the deflection of a cantilever beam

$$
y=\frac{F_{p} x^{2}}{6 E I}(3 L-x)
$$

where the origin is located at Point A, the x-axis is tangent to constant radius section of the contact surface, and $F_{p}$ is equal to

$$
F_{p}=\left(\frac{E I}{R^{\prime}}\right)\left(\frac{1}{l_{f}^{\prime}}\right)
$$

In the absence of lateral loads, the curvature of this profile matches the curvature of the noncontacting segment of the beam without exerting any force on it. When lateral loads are present this section of the contact surface prevents the beam from deflecting past its maximum rotation position. The contact surface provides a stop for the beam, limiting the lateral translation and helping to prevent buckling. Once a beam is in contact with the surface its boundary condition becomes similar to that of a fixed-pinned beam. This condition is over eight times more resistant to buckling than the fixed-free condition. A conservative approximation assumes that the load is borne by a single beam, in which case the critical load is 


$$
P_{c r}=\frac{2 \pi^{2} E I}{l_{f}^{2}}
$$

Some lateral displacement can occur when the beam is not yet in contact with the entire contact surface. The maximum lateral displacement condition, shown in Figure 9, occurs when the compressive forces cause the beam's end to slide along the cam surface to its initial position, and deflect the beam until it contacts the entire surface. The angle of rotation when this condition is possible is given by

$$
\theta_{l}=\sin ^{-1}\left\{\frac{y_{b}+l_{f}^{\prime} \sin \left[\theta_{s 2}+\tan ^{-1}\left(F_{p} l_{f}^{\prime 2} / 3 E I\right)\right]}{\delta_{c}+h / 2-R_{c}}\right\}
$$

and the resulting lateral displacement is

$$
\delta_{l}=R_{r}-\left\{x_{b}+l_{f}^{\prime} \cos \left[\theta_{s 2}+\tan ^{-1}\left(F_{p} l_{f}^{2} / 3 E I\right)\right]+\left(\delta_{c}+h / 2-R_{c}\right) \cos \theta_{l}\right\}
$$

Should the lateral displacement ever reach the maximum allowable value a secondary stop (Figure 9) is provided between the contact surface and the outside pin surface. This would prevent additional displacement, though it would also introduce friction between the two surfaces under that extreme loading condition.

\subsection{Model validation}

Validation of the PRBM analysis was done using finite element analysis (FEA), and by testing a macro-scale prototype. The torque-deflection curve for the macro-scale design was found using the PRBM analysis. The torque-deflection curve was also found using a commercial FEA program, ANSYS, as shown in Figure 10. The FEA mesh included 2671 nodes, and used the PLANE183 element, a higher order 2D, 8-node element. The PLANE 183 element has large deflection and large strain capabilities, is well suited to modeling irregular meshes, and has two degrees of freedom at each node (translation in the $x$ and $y$ directions) [15]. Two degrees of freedom were sufficient for the CCAR joint analysis, and at least two dimensions were necessary for the creation of contact elements. TARGE169 and CONTA172, 2-D contact 
elements, were used to model the interaction between the flexure and the pin, and between the flexure and the contact surface.

The flexure was constrained to have no translation at its fixed end, the contact surface was constrained to have no translation along its bottom edge, and the section of the pin was constrained to rotate about the pin's center. A force was then applied to the pin, creating a torque, and the resulting deflections and stresses were computed.

A physical prototype (Figure 11) was manufactured and tested. Figure 12 shows the test results. The PRBM and FEA predictions are very close, and have a maximum difference of only 5.4\%. More variation (up to $35.6 \%$ error) is seen in the measured values. This variation is most likely due to the variation and nonlinearity of the material properties of polypropylene and to machining tolerances.

\section{Manufacturing and assembly considerations}

Designs have been developed on three different scales. Prototypes of concepts are done on the macro scale, designs for the case study, discussed later in this paper, are done on the meso scale, and application of concepts on the micro scale have also been investigated. Table 1 lists the processes, materials, and design parameters associated with each of these scales.

Computer Numerical Control (CNC) milling was used to create macro-scale prototypes. The meso devices were designed for fabrication with micro wire electrical discharge machining (EDM) [16]. The Sandia Ultra-planar, Multi-level MEMS Technology 5 (SUMMiT V) fabrication process is the surface micromachining process used for the micro devices [17].

The minimum feature thickness and kerf width parameters constrain the geometry of a part. The thickness of a flexure, for example, cannot be less than the minimum feature thickness. Kerf width constrains the allowable distance between features of a part. Spaces between features must be greater than or equal to the kerf width. For CNC milling, the kerf width is equal to the diameter of the end mill. For micro wire EDM it is equal to the diameter of the wire plus an offset from that diameter, and can vary from 0.061 up to $0.091 \mathrm{~mm}$, depending on the thickness of the material being cut. For a $2.9 \mathrm{~mm}$ thick part (the thickness of the parts used in the CCAR joint case study) the wire kerf is estimated to be $0.076 \mathrm{~mm}$. The kerf width for the SUMMiT V 
process is not associated with a tool dimension, but is the minimum distance needed to ensure fabrication of separate features.

The minimum inside radius of a part is also constrained by the kerf width, and must be greater than or equal to one half the kerf width.

\subsection{Removed sections of a contact surface}

For a beam that is either initially straight or has a constant initial curvature, and is in contact with a surface of constant curvature, the following principle applies: if a section of the contact surface is removed, the beam segment corresponding to that section will retain the shape of the contact surface so long as a subsequent segment of the beam is still in contact with the surface.

The proof of this principle is as follows: the radius of curvature in the contacting segment is constrained to be constant, as shown in Figure 13(a). Equation (5) predicts that this constant radius of curvature will create a moment that is also constant along the segment length. For an initially straight beam ( $R_{0}=\infty$ ), equation (4) predicts that $R^{\prime}$ is equal to $R_{s}$, therefore

$$
M=\frac{E I}{R_{s}}
$$

This moment is transferred to the end of the segment corresponding to the removed contact surface, as shown in Figure 13(b). With an applied moment, the effective radius of curvature will be constant along the length of this segment of the beam. By rearranging equation (5), the effective radius of curvature of the segment is found to be

$$
R_{b}=\frac{E I}{M}
$$

Substituting equation (31) into equation (32) and simplifying gives

$$
R_{b}=R_{s}
$$

The segment of the beam corresponding to the removed contact surface has the same constant radius of curvature as the section that is in contact with the surface. 


\subsection{Manufactured configuration}

The principle of removed sections is important because the kerf widths for the $\mathrm{CNC}$ mill and micro wire EDM processes, as given in Table 1, make the CCAR joint configuration shown in Figure 1 difficult to manufacture. The spaces between the contact surfaces and the flexures are smaller than the wire kerf.

Figure 14(a) shows and alternative configuration that meets all the manufacturing constraints. All spaces between features are $0.086 \mathrm{~mm}$ or larger, the smallest inside radius is $0.043 \mathrm{~mm}$, and the minimum thickness of any feature is $0.051 \mathrm{~mm}$. Figure 14(b) shows the assembled position. The exterior flexures deflect, allowing the sections to come together and be held in place by a latch.

Once assembled, the beams and contact surfaces in this configuration have the same geometry as those of the configuration in Figure 1, except that sections of the contact surfaces are removed, as shown in Figure 15. When the beam in Figure 15(a) is in contact with the surface subsequent to the removed sections, it has the same behavior as the beam shown in Figure 15(b), where the contact surface extends to the base of the beam. The beams also have the same behavior at the beginning of rotation before either beam makes initial contact with the surfaces. The only difference between the two configurations is that the beam in Figure 15(b) will initiate contact with the surface earlier in the rotation of the joint than the beam in Figure 15(a). The behavior will vary slightly during the period.

The exterior beams connecting the sections of the CCAR joint are initially curved, and then constrained to be straight after assembly. Equation (3.4) for these beams simplifies to

$$
R_{e x t}^{\prime}=R_{0_{e x t}}
$$

where $R_{0_{e x t}}$ is the initial curvature of the exterior beams. Equation (3.11) becomes

$$
\sigma_{\max _{e x t}}=\frac{E h_{e x t}}{2 R_{0_{e x t}}}
$$

where $h_{\text {ext }}$ is the thickness of the exterior beam.

A latch holds the joint together once assembled. The beam on the latch deflects a distance of $y_{l}$ during assembly. Using linear elastic beam equations, the stress in this beam is found to be 


$$
\sigma_{\max _{\text {latch }}}=\frac{3 E y_{l} h_{l}}{2 L_{l}^{2}}
$$

where $h_{l}$ is the thickness of the latch beam, and $L_{l}$ is its length. $n_{f_{e x t}}$ and $n_{f_{\text {latch }}}$, the safety factors against yielding for the exterior beams and for the latch, respectively, can be close to one because these beams only deflect during assembly, and then are fixed in place by the mating hole for the CCAR joint.

The kerf width for the micro-scale design is small enough that the CCAR joint can be manufactured in its operational configuration.

\section{Case study}

\subsection{The Stronglink}

Sandia National Laboratory's Stronglink assembly, shown in Figure 16, is used as a case study for this research. The Stronglink is a high precision safety and arming device used to provide mechanical isolation between electrical inputs and a desired output, preventing unauthorized or unintentional detonation of a weapon [16]. The entire assembly is $30 \mathrm{~mm}$ in diameter, and future Stronglink designs will be made at even smaller scales.

The Stronglink's components include two solenoid actuated rotors, the drive rotor and the gate rotor. Both the rotors have helical extension springs to provide a restoring torque, and use rolling-element bearings to constrain lateral translation. Functional specifications for the rotors are given in Table 2 .

Components used in the Stronglink only undergo a few cycles in their life, and therefore fatigue is not a mode of failure. Due to the nature of the Stronglink's function as a safety and arming device, however, every component in the assembly must have very high reliability. Because of the assembly methods required to install them, the helical springs create a reliability concern. The springs have wire diameters ranging from 50 to 100 micrometers. The helical coils are wound using semi-automated production equipment. The hooks at the end of the springs, however, are formed by hand with tweezers and pliers, and the springs are manually assembled into the Stronglink. These manual operations can nick or flatten the wire, creating areas of high stress that may lead to failure [16]. 
The bearings used in the Stronglink are the smallest commercially available miniature bearings with an outer diameter of $2.54 \mathrm{~mm}$. As the size of the Stronglink decreases in future designs, the size of the bearings are not able to decrease with it. Alternative solutions are needed, and the CCAR joint is an ideal candidate for replacing both the bearings and helical springs with a single part.

\subsection{Case study results}

Table 3 lists the dimensions used for the drive and gate rotor meso-scale designs, and Figure 17 shows the scaled polypropylene prototypes of these designs. The drive rotor CCAR joint has more flexures to increase its torque, and the gate rotor design has less in order to increase its rotation.

Table 4 lists the results. The nominal torque curves closely match the torque requirements. The nominal maximum torque is equal to the desired maximum torque for both the designs. The nominal minimum torque for the drive rotor is $98 \%$ of the desired value, and for the gate rotor it is $94 \%$ of the desired value. The upper and lower bounds for the maximum torque range are calculated based on a surface profile tolerance of $\pm 0.00508 \mathrm{~mm}$, as listed in Table 2 . They range from $56 \%$ to $194 \%$ of the desired value for the drive rotor, and $44 \%$ to $195 \%$ of the desired value for the gate rotor.

Figures 18 and 19 show the nominal torque-deflection curve as well as the lower and upper bounds for the torque. As the flexures contact the adjacent contact surfaces, the torque-deflection relationship becomes very nonlinear. In these designs, however, the radii of the contact surfaces were decreased, resulting in the flexures contacting a smaller percentage of the surface at the maximum rotation of the joint (indicated by the "\% of Surface Contacted" values in Table 4). The torque curves, therefore, are more linear. This is necessary because the flexures always contact a higher percentage of the surface in the upper bound condition than in the nominal condition. If the flexures contact a high percentage of the surface in the nominal condition, then the upper bound condition flexures will contact even more of the surface and cause the torque curve to become very nonlinear, resulting in very high torque.

If the flexures in the upper bound condition contact the entire surface before reaching the maximum rotation position, then the joint will be unable to complete its specified rotation. This is illustrated in Figure 20. The design used in Figure 20 has a large contact surface radius, and in the nominal condition the flexures 
contact $66 \%$ of the surface at the maximum rotation of 22.85 degrees. In the upper bound condition, however, the flexure contacts $100 \%$ of the surface at only 21.35 degrees of rotation. The torque is very high, and the joint is unable to rotate the full 22.85 degrees.

This problem is avoided by lowering the contact surface radius in the drive and gate rotor designs. The cost of doing so is that the safety factors are lowered as well. The lowest safety factor is in the upper bound condition of the drive rotor, which has a safety factor against yielding that is just above 1. Exceeding the yield strength of the material, however, would only result in some local deformation in the flexures, and not in catastrophic failure. Flexure deformation would lower the output torque of the joint. In the upper bound condition this would bring the output torque closer to the desired torque values, and would not be detrimental to the design. The lower bound condition, where decreased torque would be most problematic, also has the highest safety factor.

Table 4 lists the values for $\delta_{l}$, the maximum lateral displacement. These values do not represent the actual displacement due to the lateral loads given in Table 2, but represent the maximum possible displacement before the contact surfaces constrain further deflection and lateral displacement. The actual displacement is most likely less than this value. The drive rotor joint has a nominal maximum lateral displacement equal to the maximum allowable displacement specified in Table 2. The nominal maximum lateral displacement for the gate rotor joint is almost twice the maximum allowable value.

Should the actual lateral displacement reach the maximum allowable value, the secondary stop between the outer surface of the pin and the inner surface of the joint would prevent further displacement. The nominal distance between these two surfaces is equal to the maximum allowable displacement, $0.0254 \mathrm{~mm}$. If the surfaces contact, some of the load would be transferred from the flexures to the surface, constraining further deflection but introducing some friction.

\section{Conclusions}

A unique joint capable of replacing both bearings and springs has been presented, and a model for predicting its behavior developed and validated. The CCAR joint can be made using planar fabrication methods, and is easily scaled. By the Moon et al. criteria [3] discussed earlier, the CCAR joint rates "good" in 
all five criteria. The contact interactions in the CCAR joint increase the maximum rotation of the joint and increase the lateral stiffness, while allowing the joint to remain compact enough to fit within roughly the same space as a bearing. Axis drift is minimal, and the maximum stress is evenly distributed along the contacting segment of the flexures, so there are no stress concentrations.

The CCAR joint is able to meet the demanding design constraints of the Stronglink case study. The nominal torque values closely match the torque requirements, the nominal lateral deflection is within the allowable range, and the available design space is not exceeded. Nominal safety factors against yielding are 1.15 for the drive rotor, and 1.39 for the gate rotor.

\section{Acknowledgements}

Funding from Sandia National Laboratories is gratefully acknowledged, as is the support from Dr. Greenwood and Sandia personnel.

Other individuals that deserve acknowledgement for their contributions to this work are Craig Lusk, who was closely involved with the development of concepts and of analytical models, and Daniel Wilcox's, whose ideas were instrumental in developing the approach for the fabrication of the CCAR joint.

\section{References}

[1] Norton, R. L., Machine Design, Prentice-Hall, Upper Saddle River, NJ, 1998

[2] Howell, L. L., Compliant Mechanisms, John Wiley \& Sons Inc., NY, 2001

[3] Moon, Y., Trease, B. P., and Kota, S., Design of Large-Displacement Compliant Joints. Proceedings of the 2002 Design Engineering Technical Conferences, DETC2002/MECH-34207

[4] Paros, J. M., and Weisbord, L., How to Design Flexure Hinges. Machine Design, Nov. 25, 1965, pp. $151-156$

[5] Kyusojin, A., and Sagawa, D., Development of Linear and Rotary Movement Mechanisms by Using Flexible Strips. Bulletin of Japan Society of Precision Engineering, Vol. 22, No. 4, Dec., 1988, pp. 309-314 
[6] Bona, F., and Zelenika, S., Precision Positioning Devices Based on Elastic Elements: Mathematical Modeling and Interferometric Characterization. Seminar on Handling and Assembly of Microparts, Vienna, Nov., 1994

[7] Smith, S., Flexures, Elements of Elastic Mechanisms, Gordon and Breach Science Publishers, 2000

[8] Weinstein, W. D., Flexural Pivot Bearings. Machine Design, June 10, 1965, pp. 150-157

[9] Goldfarb, M., and Speich, J. E., A Well-Behaved Revolute Flexure Joint for Compliant Mechanism Design. Journal of Mechanical Design, Trans. ASME, Vol. 121, No. 3, 1999, pp. 424-429

[10] Mankame, N. D., Ananthasuresh, G. K., Contact Aided Compliant Mechanisms: Concept and Preliminaries. Proceedings of the 2002 ASME Design Engineering Technical Conferences, DETC2002/MECH-34211

[11] Guérinot, A. E., Compliant Mechanisms Subjected to Compressive Loads. M. S. Thesis, Brigham Young University, Provo, UT, 2003

[12] Votta, F.A., Theory and Design of Long-deflection Constant Force Spring Elements. Transaction of the ASME, Vol. 74, 1952, pp. 439-450

[13] Chironis, N. P., and Sclater, N., Mechanisms and Mechanical Devices Sourcebook, 2nd Ed., McGrawHill, NY, 1996

[14] Benavides, G.L., Bieg, L.F., Saavedra, M.P., and Bryce, E.A., High Aspect Ratio Meso-Scale Parts Enabled by Wire Micro-EDM. Microsystems Technologies Journal, Springer-Verlag, Vol. 8, Number 6, Sept., 2002

[15] ANSYS Release 8.0 Documentation, 2003, ANSYS Element Ref.> Element Library> PLANE183, SAS IP Inc

[16] Kennedy, J. A., Compliant, In-Plane Ratchet and Pawl Micromechanisms for Safety Applications. M.S. Thesis, Brigham Young University, Provo, UT, 2003

[17] http://www.sandia.gov/mstc/technologies/micromachines/tech-info/technologies/summit5.html 
Table 1. Manufacturing processes and parameters.

\begin{tabular}{llll}
\hline Scale & Macro & Meso & Micro \\
\hline Process & CNC Milling & Micro Wire EDM & SUMMiT V \\
Material & Polypropylene & Titanium 6Al 4V & Polycrystalline Silicon \\
E [GPa (Mpsi)] & $1.4(0.2)$ & $114(16.5)$ & $169(24.5)$ \\
$\mathrm{S}_{\mathrm{y}}[\mathrm{MPa}(\mathrm{kpsi})]$ & $34(5)$ & $825(120)$ & $1800(261)$ \\
Min. Feature Thickness [mm (in)] & - & $0.0508(0.002)$ & $0.001(0.000039)$ \\
Kerf Width [mm (in)] & $2.38(0.0938)$ & $0.0762(0.003)$ & $0.001(0.000039)$ \\
Surface Profile Tolerance [mm (in)] & - & $\pm .00508(0.0002)$ & - \\
\hline
\end{tabular}

Table 2. Functional specifications for the Stronglink case study.

\begin{tabular}{ccc}
\hline & Drive Rotor & Gate Rotor \\
\hline Lateral Load & $3.55 \mathrm{~N}$ & $0.089 \mathrm{~N}$ \\
Max. Lateral Displacement & $0.025 \mathrm{~mm}$ & $0.025 \mathrm{~mm}$ \\
Rotation & 8 degrees & 15 degrees \\
Min. Torque & $0.00154 \mathrm{~N}-\mathrm{m}$ & $0.000116 \mathrm{~N}-\mathrm{m}$ \\
Max. Torque & $0.00307 \mathrm{~N}-\mathrm{m}$ & $0.000232 \mathrm{~N}-\mathrm{m}$ \\
\hline
\end{tabular}

Table 3. Design dimensions (all values are in $\mathrm{mm}$ ).

\begin{tabular}{llllllllll}
\hline & $R r$ & $R p$ & $R c$ & $R s$ & $\delta c$ & $h$ & $b$ & $r Q$ & $n$ \\
\hline Drive Rotor & 1.27 & 0.483 & 0.0826 & 6.41 & 0.265 & 2.07 & 2.54 & 0.0838 & 6 \\
Gate Rotor & 1.27 & 0.483 & 0.140 & 4.86 & 0.265 & 0.0508 & 1.32 & 0.0559 & 4 \\
\hline
\end{tabular}


Table 4. (a) Drive rotor results, and (b) gate rotor results.

(a)

\begin{tabular}{|c|c|c|c|c|c|c|c|}
\hline & $\begin{array}{l}\text { Min } \\
\text { Torque } \\
(\mathrm{N}-\mathrm{m})\end{array}$ & $\begin{array}{l}\text { Max } \\
\text { Torque } \\
(\mathrm{N}-\mathrm{m})\end{array}$ & $\begin{array}{c}\text { Minimum } \\
\text { Rotation } \\
\text { Position (deg) }\end{array}$ & $\begin{array}{c}\text { Maximum } \\
\text { Rotation } \\
\text { Position (deg) }\end{array}$ & $\begin{array}{c}\% \text { of } \\
\text { Surface } \\
\text { Contacted }\end{array}$ & $\begin{array}{c}\delta_{l} \\
(\mathrm{~mm})\end{array}$ & $\begin{array}{l}\text { Safety } \\
\text { Factor }\end{array}$ \\
\hline Nominal & 0.00150 & 0.00307 & 14.85 & 22.85 & 26.0 & 0.0044 & 1.15 \\
\hline Upper & 0.00260 & 0.00595 & 14.85 & 22.85 & 64.4 & 0.0027 & 1.02 \\
\hline Lower & 0.000689 & 0.00172 & 14.85 & 22.85 & 0 & 0.0062 & 1.35 \\
\hline & $\begin{array}{l}\text { Min } \\
\text { Torque } \\
(\mathrm{N}-\mathrm{m})\end{array}$ & $\begin{array}{l}\text { Max } \\
\text { Torque } \\
(\mathrm{N}-\mathrm{m})\end{array}$ & $\begin{array}{c}\text { Minimum } \\
\text { Torque } \\
\text { Position (deg) }\end{array}$ & $\begin{array}{c}\text { Maximum } \\
\text { Torque } \\
\text { Position (deg) }\end{array}$ & $\begin{array}{c}\% \text { of } \\
\text { Surface } \\
\text { Contacted }\end{array}$ & $\begin{array}{c}\delta_{l} \\
(\mathrm{~mm})\end{array}$ & $\begin{array}{l}\text { Safety } \\
\text { Factor }\end{array}$ \\
\hline Nominal & 0.000110 & 0.000232 & 32 & 47 & 0 & 0.048 & 1.39 \\
\hline Upper & 0.000216 & 0.000452 & 32 & 47 & 18.4 & 0.036 & 1.17 \\
\hline Lower & 0.0000452 & 0.000102 & 32 & 47 & 0 & 0.061 & 1.99 \\
\hline
\end{tabular}




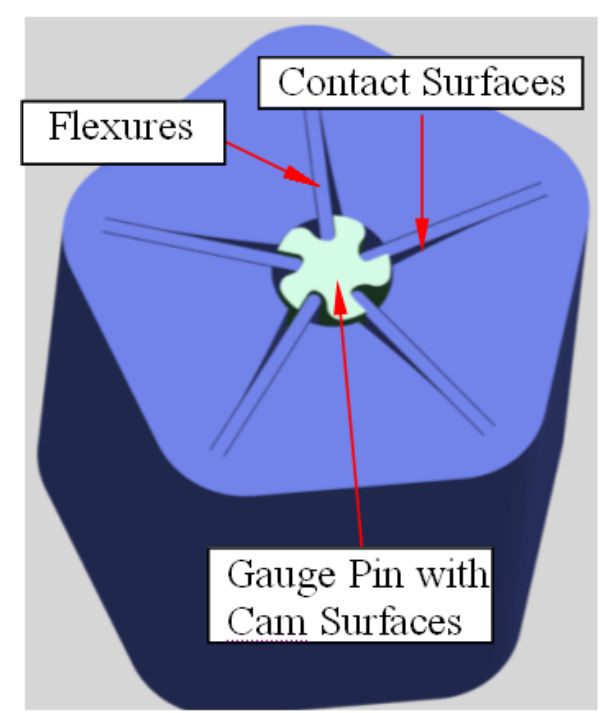

(a)

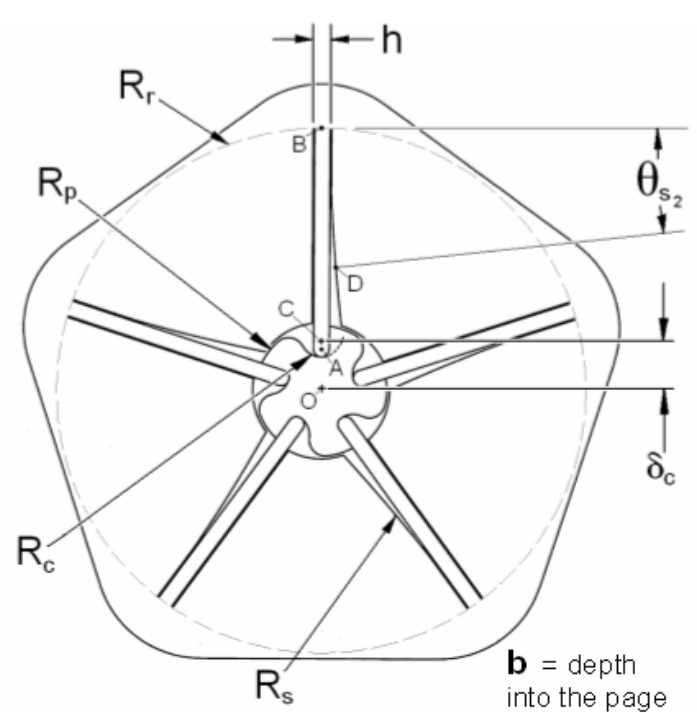

(b)

Figure 1. (a) CCAR joint design and (b) design parameters.

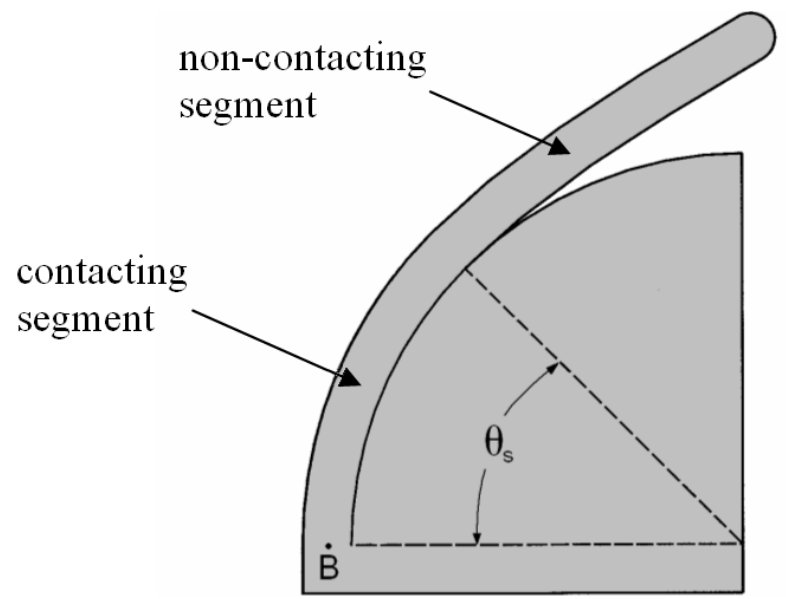

Figure 2. A beam in contact with a surface of constant radius. 


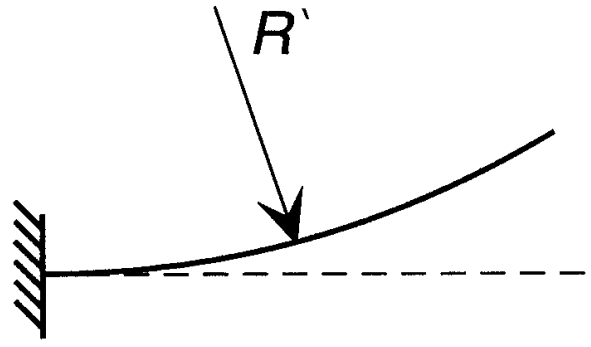

(a)

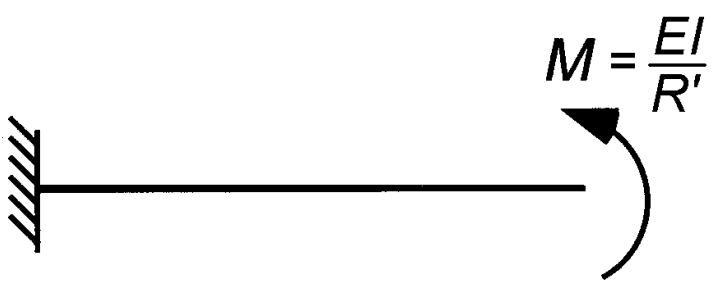

(b)

Figure 3. (a) Beam constrained by contact surface and (b) its equivalent beam with an applied moment. (Adapted from [])

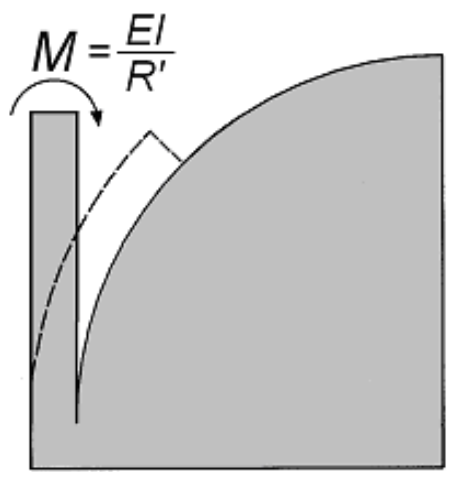

Figure 4. Segment with curvature constrained by the contact surface (dashed lines) and an equivalent beam with an applied moment.

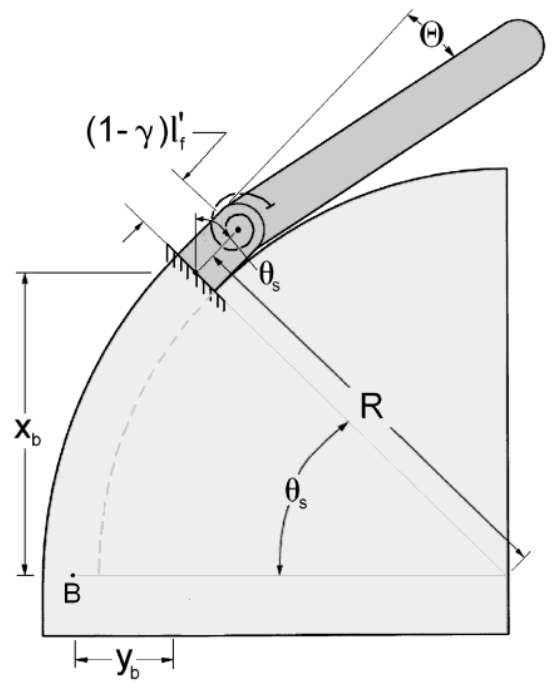

Figure 5. Pseudo-rigid-body model of beam. 


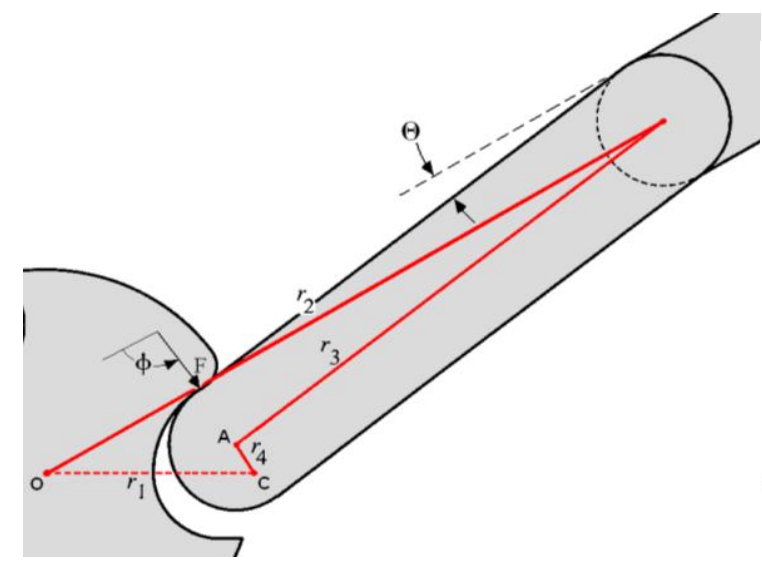

(a)

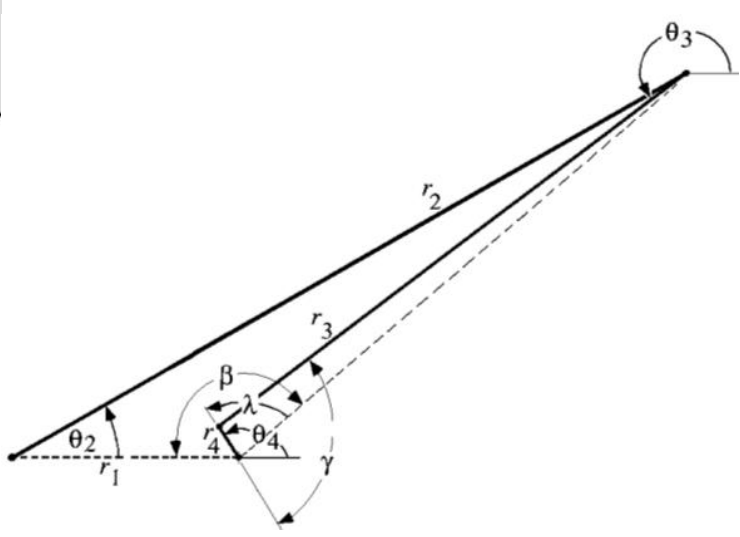

(b)

Figure 6. (a) Non-contacting segment of beam and gauge pin, and (b) equivalent rigid-link four-bar mechanism.

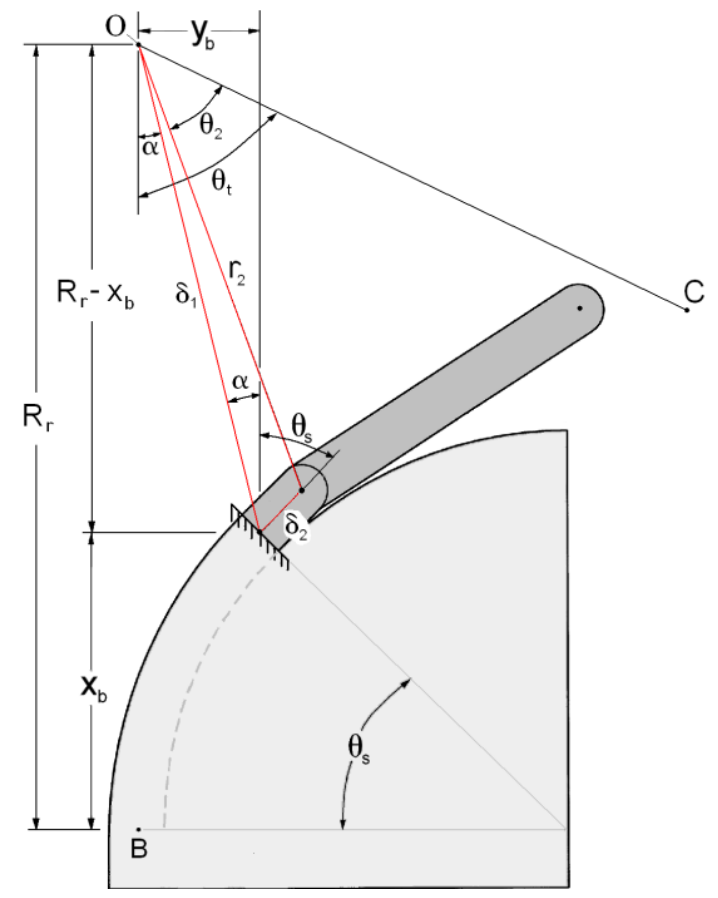

Figure 7. Kinematic analysis parameters. 


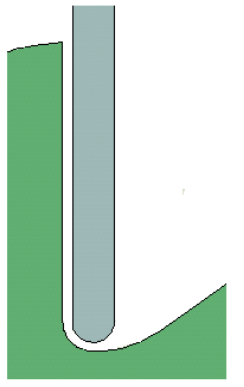

(a)

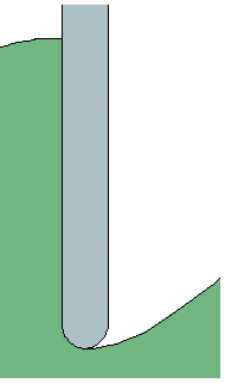

(b)

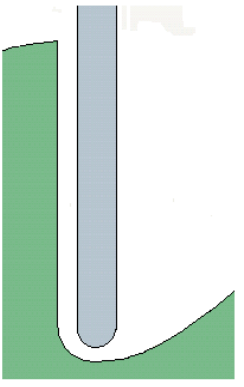

(c)

Figure 8. Flexure end and cam surface on pin in the (a) nominal condition, (b) upper bound condition, and (c) lower bound condition.

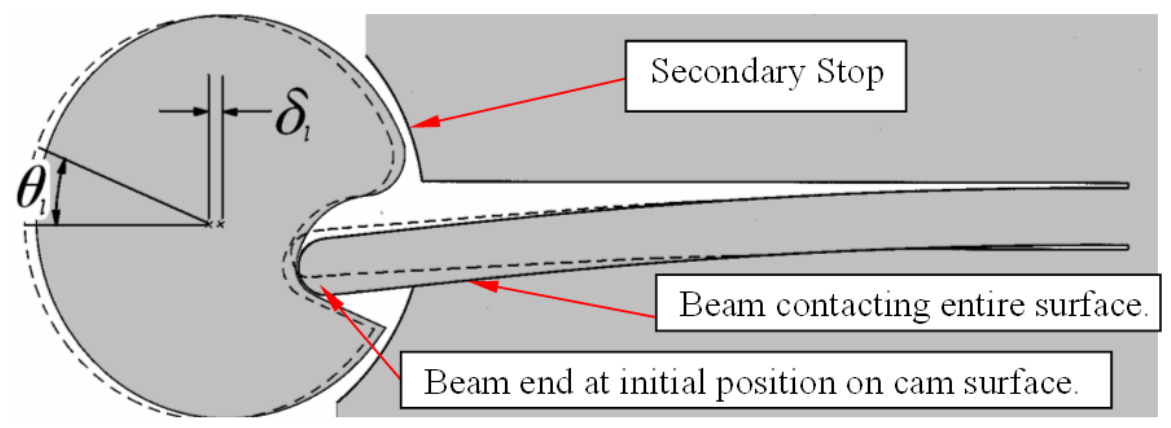

Figure 9. Normal position (dashed lines) and maximum displacement position.

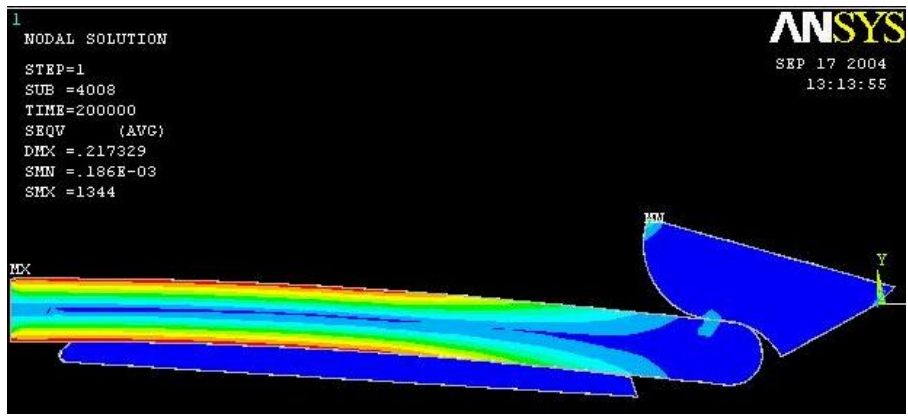

Figure 10. FEA model with contact elements. 


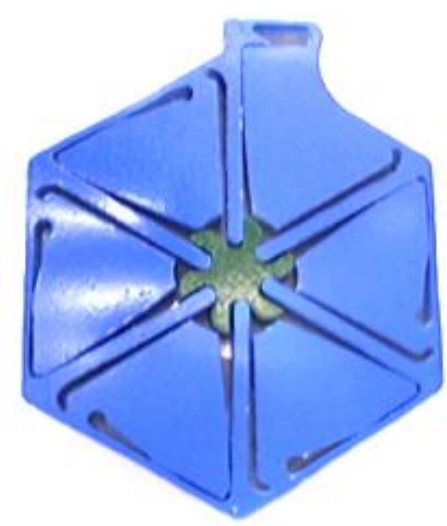

Figure 11. Prototype of the CCAR joint.

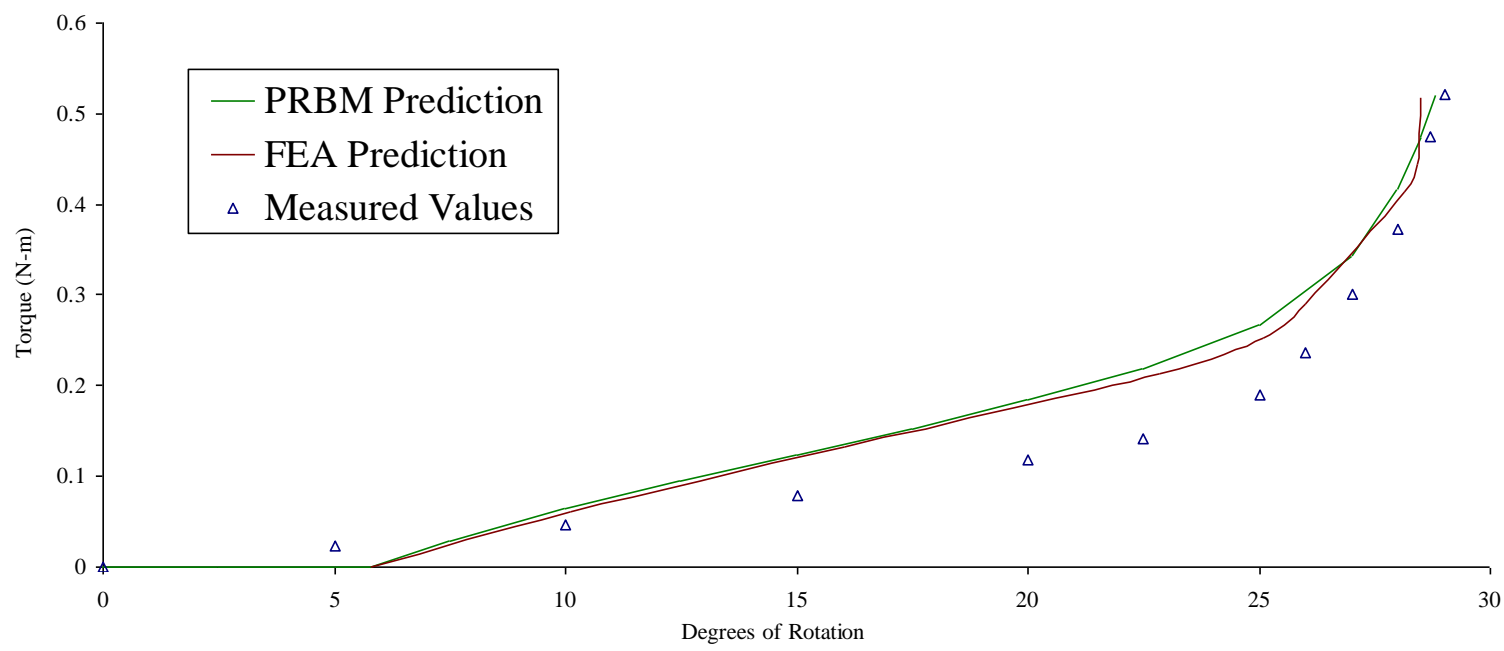

Figure 12. Results of PRBM predictions, FEA predictions, and prototype testing.

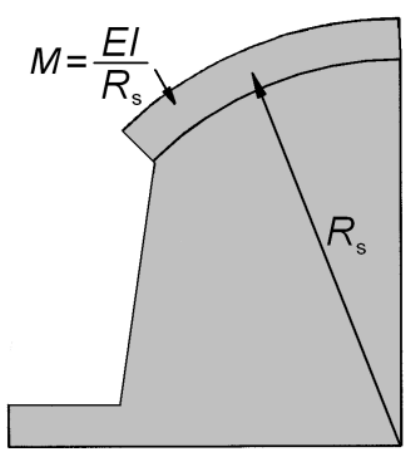

(a)

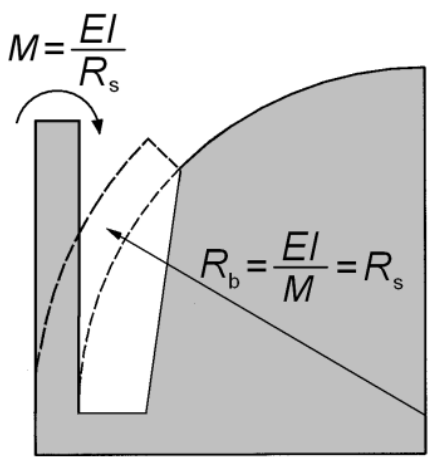

(b)

Figure 13. (a) Segment of beam in contact with surface, and (b) segment of beam corresponding to removed contact surface. 


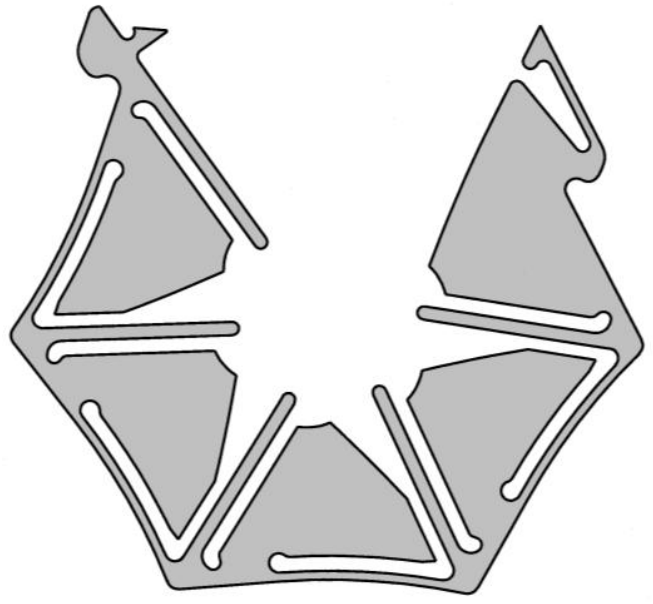

(a)

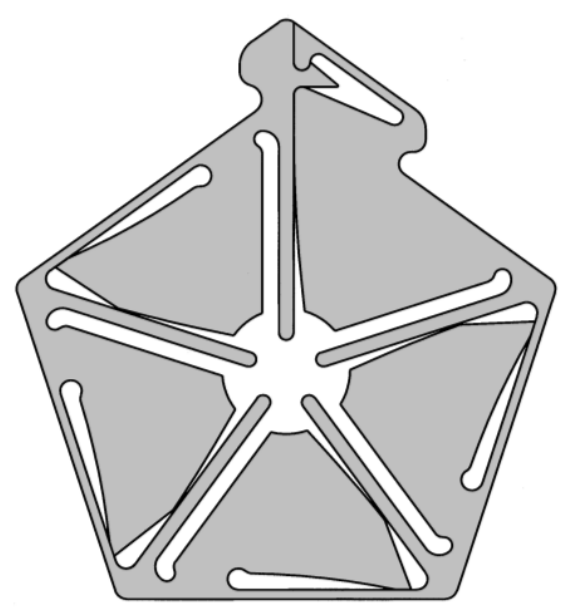

(b)

Figure 14. (a) Manufactured position and (b) assembled position of CCAR joint.

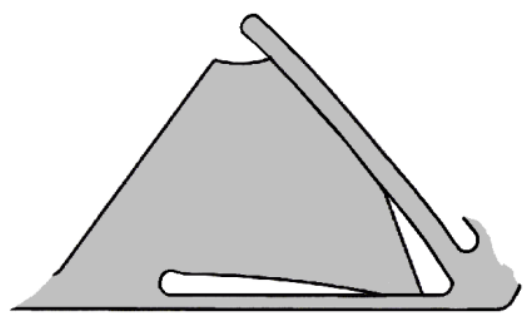

(a)

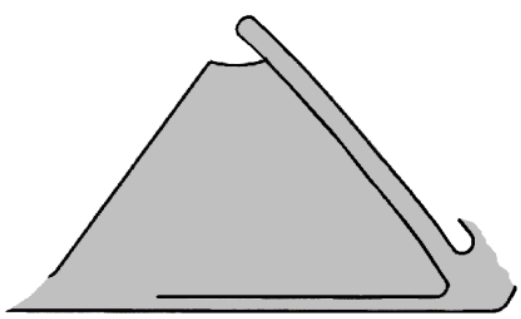

(b)

Figure 15. (a) Section of joint with cuts, and (b) equivalent section without cuts.

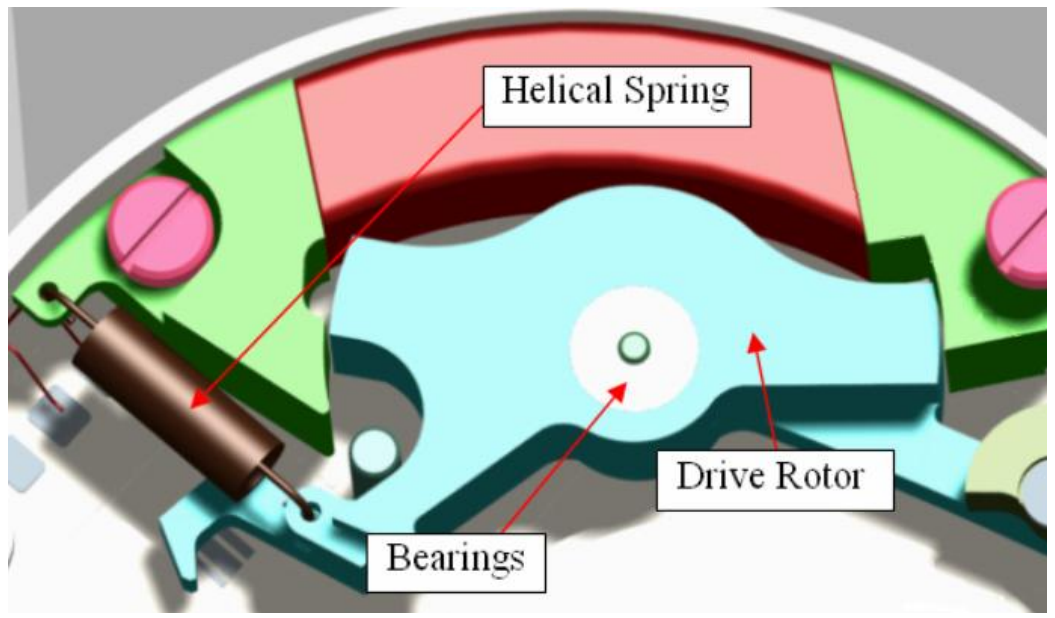

Figure 16. Drive components in the Stronglink assembly. 


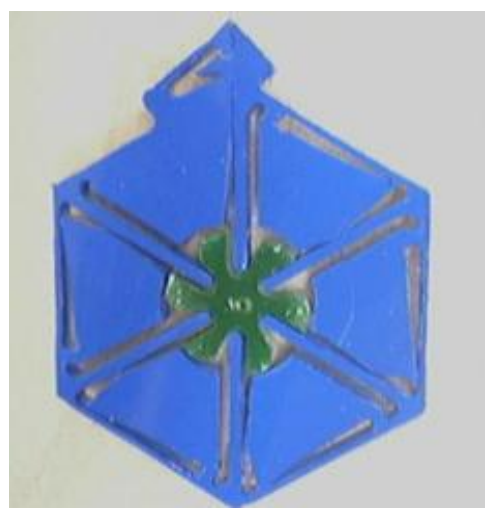

(a)

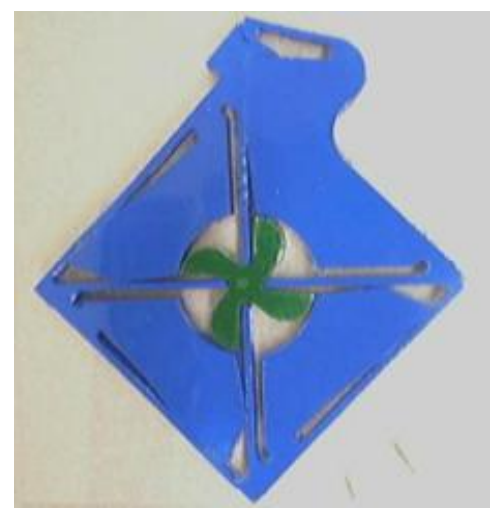

(b)

Figure 17. Prototypes of the (a) drive rotor CCAR joint, and (b) gate rotor CCAR joint.

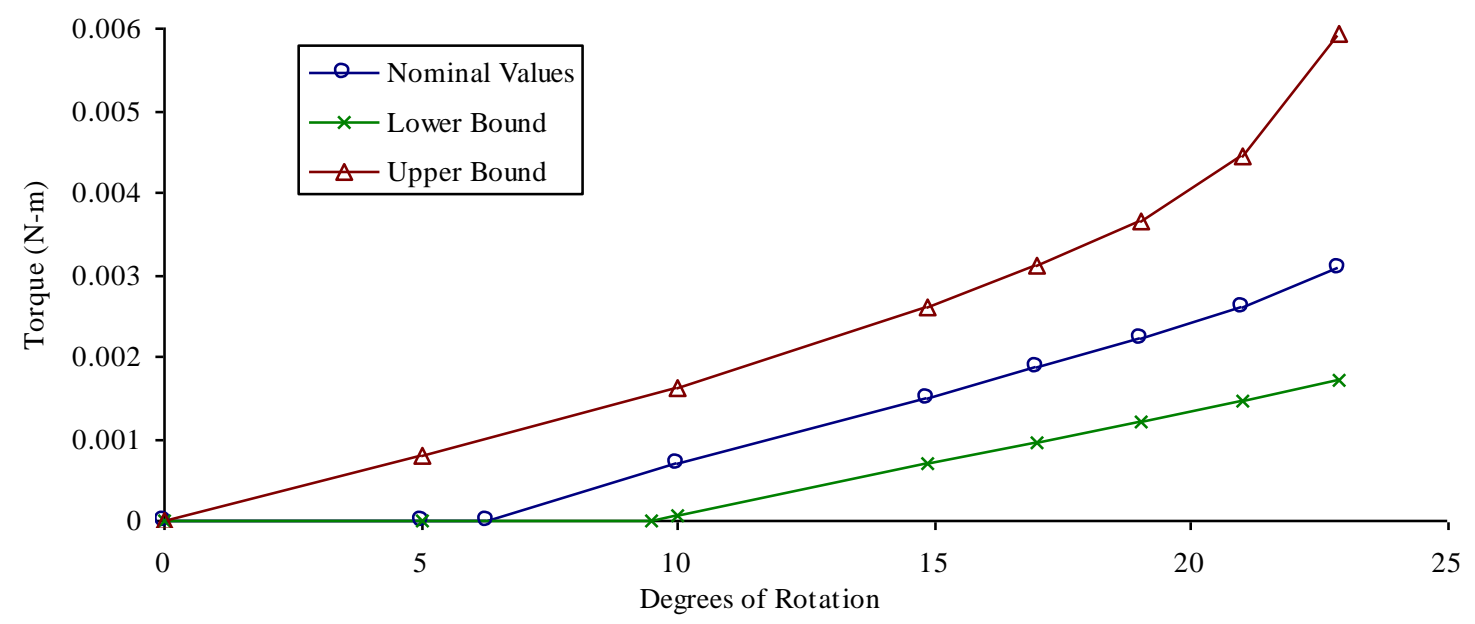

Figure 18. Effect of tolerances on the drive rotor CCAR joint.

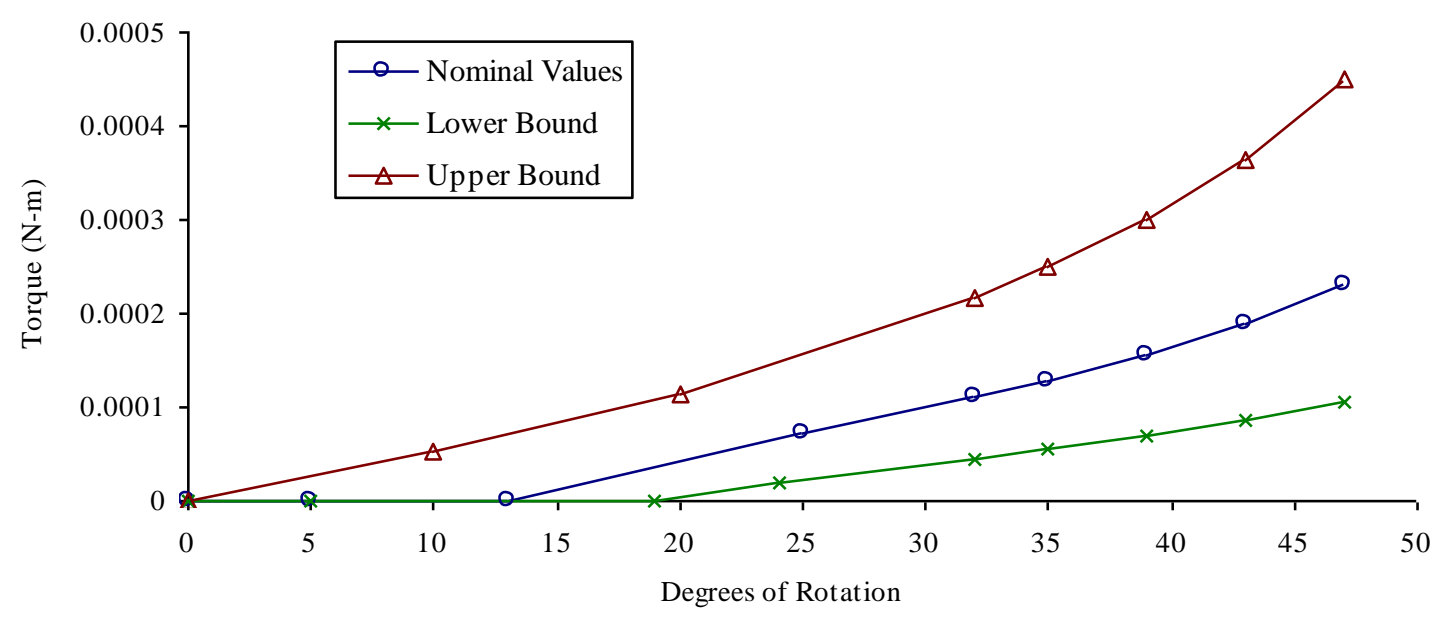

Figure 19. Effect of tolerances on the gate rotor CCAR joint. 


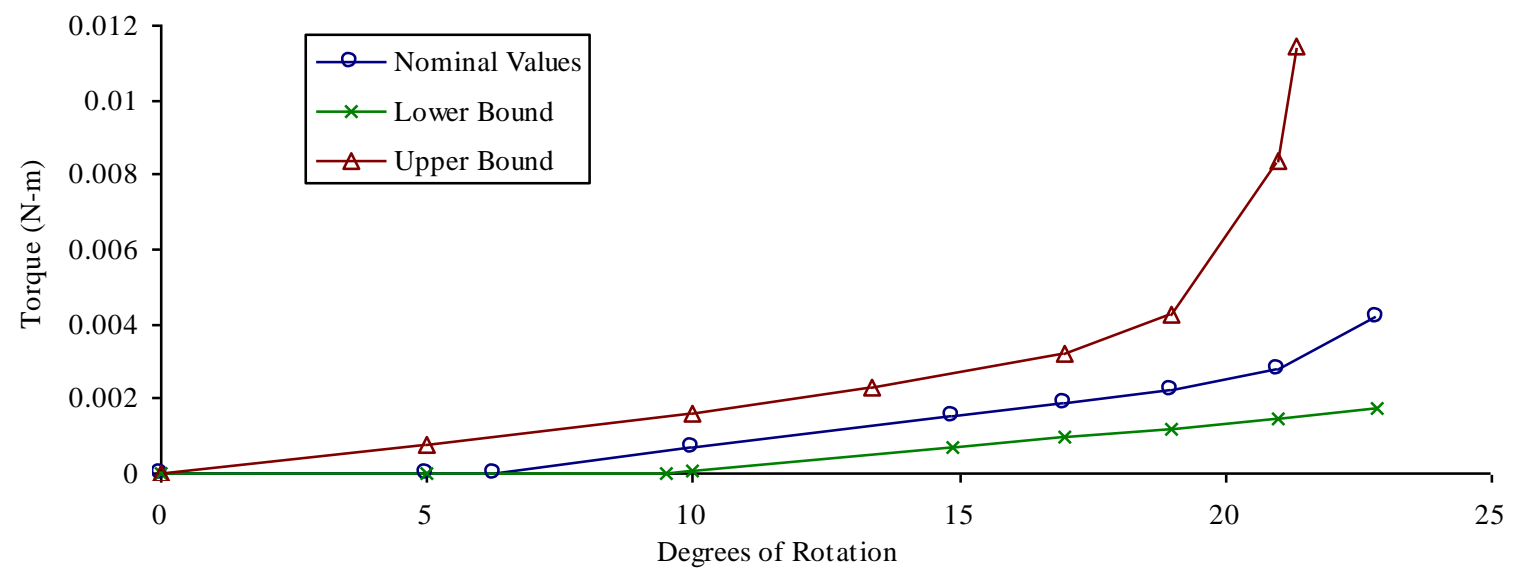

Figure 20. Design with high percentage of flexure length in contact with surface. 